\title{
Molecular Diagnostics Laboratory Shared Resource
}

National Cancer Institute

\section{Source}

National Cancer Institute. Molecular Diagnostics Laboratory Shared Resource. NCI

Thesaurus. Code C39460.

The Molecular Diagnostics Laboratory Shared Resource provides support to Cancer Center investigators with specimen processing and tracking, DNA sequencing, microsatellite and SNP analyses, quantitative PCR, protein analysis as well as other services, to analyze genes and genes products that are, or may be, involved in the etiology of cancer. 